
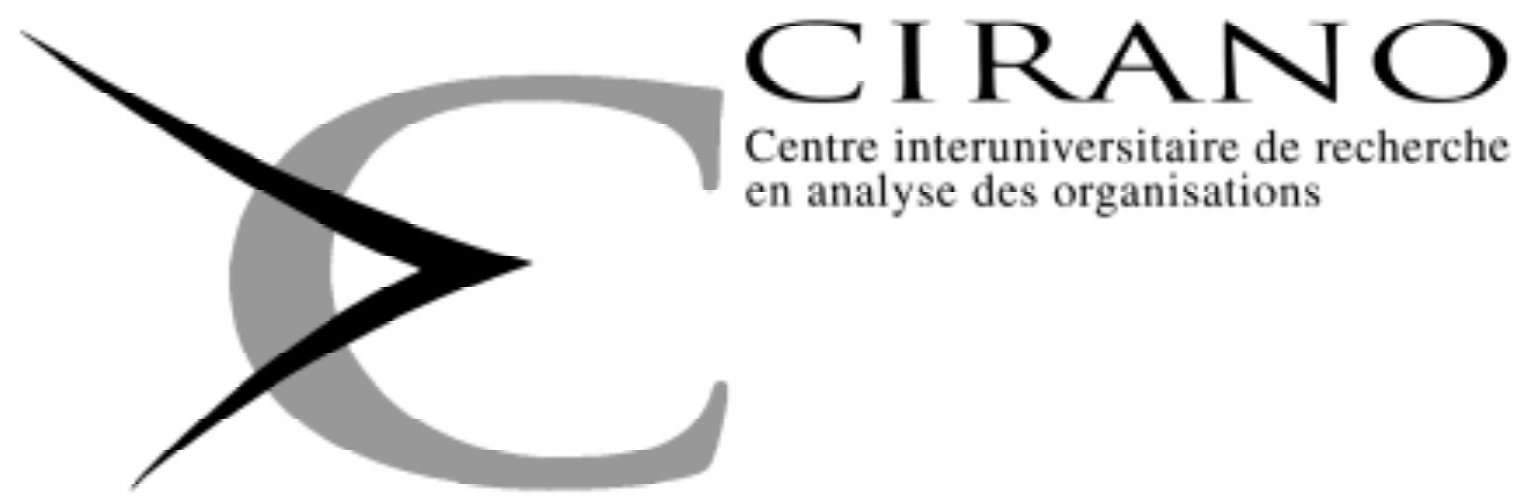

Centre interuniversitaire de recherche en analyse des organisations

Série Scientifique

Scientific Series

95s-37

DYNAMIC INCENTIVE

CONTRACTS WITH

UNCORRELATED PRIVATE

INFORMATION AND HISTORY

DEPENDENT OUTCOMES

Gérard Gaudet, Pierre Lasserre, Ngo Van Long 


\section{CIRANO}

Le CIRANO est une corporation privée à but non lucratif constituée en vertu de la Loi des compagnies du Québec. Le financement de son infrastructure et de ses activités de recherche provient des cotisations de ses organisations-membres, d'une subvention d'infrastructure du ministère de l'Industrie, du Commerce, de la Science et de la Technologie, de même que des subventions et mandats obtenus par ses équipes de recherche. La Série Scientifique est la réalisation d'une des missions que s'est données le CIRANO, soit de développer l'analyse scientifique des organisations et des comportements stratégiques.

CIRANO is a private non-profit organization incorporated under the Québec Companies Act. Its infrastructure and research activities are funded through fees paid by member organizations, an infrastructure grant from the Ministère de l'Industrie, du Commerce, de la Science et de la Technologie, and grants and research mandates obtained by its research teams. The Scientific Series fulfils one of the missions of CIRANO: to develop the scientific analysis of organizations and strategic behaviour.

\section{Les organisations-partenaires / The Partner Organizations}

-Ministère de l'Industrie, du Commerce, de la Science et de la Technologie.

-École des Hautes Études Commerciales.

-École Polytechnique.

-Université de Montréal.

-Université Laval.

-McGill University.

-Université du Québec à Montréal.

-Bell Québec.

-La Caisse de dépôt et de placement du Québec.

-Hydro-Québec.

-Fédération des caisses populaires de Montréal et de l'Ouest-du-Québec.

-Téléglobe Canada.

-Société d'électrolyse et de chimie Alcan Ltée.

-Avenor.

-Service de développement économique de la ville de Montréal.

Ce document est publié dans l'intention de rendre accessible les résultats préliminaires de la recherche effectuée au CIRANO, afin de susciter des échanges et des suggestions. Les idées et les opinions émises sont sous l'unique responsabilité des auteurs, et ne représentent pas nécessairement les positions du CIRANO ou de ses partenaires.

This paper presents preliminary research carried out at CIRANO and aims to encourage discussion and comment. The observations and viewpoints expressed are the sole responsibility of the authors. They do not necessarily represent positions of CIRANO or its partners.

\section{ISSN 1198-8177}




\title{
Dynamic Incentive Contracts with Uncorrelated Private Information and History Dependent Outcomes*
}

\author{
Gérard Gaudet ${ }^{\dagger}$, Pierre Lasserre ${ }^{\dagger}$, Ngo Van Long ${ }^{\dagger}$
}

\section{Résumé / Abstract}

\begin{abstract}
La dynamique des modèles actuels de contrats incitatifs provient de la capacité du principal, à partir des actions observées dans le présent, d'apprendre quelque chose sur l'information qui ne lui sera pas directement accessible dans le futur. Nous étudions ici une autre source de dynamique, négligée dans la littérature sur l'information mais standard dans toutes les autres branches de la science économique, et qui résulte du fait que les actions courantes définissent les opportunités futures. C'est ce qui se passe lorsqu'il y a investissement, apprentissage, R-D, etc. Pour bien identifier les propriétés dynamiques résultant de ce type de situations dans les modèles de principal-agent avec information asymétrique, nous nous en tenons à des modèles où il n'y a aucune corrélation entre information présente et information future, si bien qu'aucun effet dynamique ne résulte directement de l'asymétrie d'information.

In existing papers on dynamic incentive contracts, the dynamic structure of the principal-agent relationship arises exclusively from the ability of the principal to learn about the hidden information over time. In this paper we deal with a different source of dynamics, which is considered standard in all areas of economics other than the information literature: we study situations where current opportunities depend on past and current actions, notwithstanding any information conveyed by the actions. Standard examples include investment, "Learning by doing", and R\&D. In order to focus on this neglected source of dynamics, we restrict our attention to situations involving asymmetric information in each period, but without any intertemporal informational correlation, so that no dynamic effect arises from informational asymmetries directly. This makes comparisons with static results both easier and more interesting.
\end{abstract}

Key words: Incentive contracts, Dynamic, Asymmetric information, Principal-agent relationship, Investment, Learning by doing.

Mots clés: Contrats incitatifs, Dynamique, Information asymétrique, Relation principal-agent, Investissement, Learning by doing.

Address. Please address all correspondence to Pierre Lasserre, Département des sciences économiques, UQAM, C.P. 8888, succ. A, Montréal (Qc), Canada, H3C 3P8. E-mail: pierre@ uqam.ca.

\footnotetext{
- Financial support from the Fonds FCAR du Gouvernement du Québec is gratefully acknowledge.

${ }^{\dagger}$ Département des sciences économiques, Université du Québec à Montréal and CIRANO.

‡ Department of Economics, McGill University and CIRANO.
} 


\section{Introduction}

When studying dynamic incentive contracts $\Gamma$ authors have focused their attention on a repeated relationship in which the principal was potentially able to become better informed as the relationship evolved over time. In that literature $\Gamma$ in each period $\Gamma$ once the hidden information has been revealed to the agent $\Gamma$ the surplus to be shared between the principal and the agent depends on current actions only; thus the dynamic structure of the relationship arises exclusively from the ability of the principal to learn about the hidden information over time.

If there is no intertemporal correlation between realizations of the private information $\Gamma$ the principal does not learn anything about the future from the revelation of current information; as a result the dynamic relationship is a repetition of static incentive contracts based on the revelation principle. If $\Gamma$ instead $\Gamma$ the current realization carries information about the probability distribution of future realizations revelation by the agent of current information affects future relationships $\Gamma$ opening the way to a ratchet effect. Principal-agent contractual relationships have been studied extensively in that context under various conditions regarding commitment Trenegotiation Tand the duration of the relationship. (see Besanko and SappingtonГ1987; Laffont and TiroleГ 1988; Baron 1989$)$. The major results sharply contrast with the static principal-agent theory. In particular $\Gamma$ in the absence of commitment $\Gamma$ the revelation principle breaks down; there does not exist any separating equilibria if there is a continuum of types; "good" types are not preserved from distorting contract requirements.

In this paper $\Gamma$ we deal with a different source of dynamics $\Gamma$ the one which is considered standard in all areas of economics other than the information literature. We envisage situations where current opportunities depend on past $\Gamma$ as well as current $\Gamma$ actions $\Gamma$ notwithstanding any information conveyed by the actions. Standard examples include investment $\Gamma^{\text {"Learning by doing" Texhaustible-resource extraction } \Gamma a n d ~ R ~ \& ~ D . ~}$ 
Substantial theoretical and empirical work on these topics has been carried out over the years Tincluding setups involving uncertainty and strategic opportunities. Surprisingly the theory has never been extended to deal with information asymmetries. Although our paper may be viewed as a first step toward fulfilling this need $\Gamma$ it is also a contribution to the principal-agent literature. Indeed our focus is on the robustness of some results of the static principal-agent literature to the introduction of the dynamic features mentioned above. While we use a simple $\Gamma$ specific $\Gamma$ model to help intuition $\Gamma$ our points are fairly general. In particular we do not assume Markovian characteristics $\Gamma$ although this is the prevalent approach in the literature on investment extraction Tetc. Tand we do not impose a fixed optimization period $\Gamma a s$ is usually done in dynamic principal-agent models.

In order to focus on the dynamics that result directly from observable actions Trather than from informational asymmetries Twe assume uncorrelated realizations of the hidden factor over time; to facilitate interpretation we also assume that the hidden factor is a characteristic of the agent $\Gamma$ i.e. we analyze adverse selection problems. Thus the principal does not learn anything about the future from the revelation of the current realization. Under such circumstances Tit is obvious Tas illustrated by Besanko and Sappington (1987Г section 7) $\Gamma$ that the revelation principle remains effective. This makes comparisons with static results both easier and more interesting.

The following results apply to such a wide class of models that they can be taken to summarize conventional wisdom about static adverse-selection principal-agent models.

Fact 1 Under symmetric information about the agent's type The agent obtains no rent and its activity is optimal.

Fact 2 The "worst" type that is willing to participate receives no rent.

Fact 3 There is no distortion to the "best" type. 
Fact 4 Under standard technical assumptions that include a monotonic hazard rate for the distribution of types $\Gamma$ the principal's problem has a unique interior solution; this solution is separating for all types that participate $\Gamma$ distorting except for the best type Tand such that the agent's action Tand its rent Tare monotonic functions of its type.

Under the class of dynamic setups just outlined $\Gamma$ we will show that $\Gamma$ with the trivial exception of Fact 1 Th these results have to be modified in general. In particular $\Gamma$ we will emphasize what happens to Facts 2 and $3 \Gamma$ and why. In Section $2 \Gamma$ after underlining the scope of our discussion by providing a few examplesTwe will present a model and describe its main features under symmetric and then under asymmetric information. In particular Tit will be shown that the absence of commitment requires the imposition of an additional participation constraint $\Gamma$ not present in static models. Then Section 3 will provide a more thorough comparison of the static $\Gamma$ and the dynamic $\Gamma$ results $\Gamma$ focusing in particular on the "best type". By thenTit should be clear to the reader that Facts $2-4$ are specific to static situations $\Gamma$ and why. The example provided in Section 4 Therefore is more than a simple illustration; it emphasizes that exceptions may arise under assumptions that might be considered benign in the asymmetric information literature.

\section{The model}

\section{$2.1 \quad$ Setup}

In order to help intuition let us think of a principal-agent relationship where the principal is a government seeking to appropriate itself a share of excess profits by its agent. The latter is a firm whose technology is such that current profits depend on past and current levels $q_{1}, q_{2}, \ldots, q_{t}$ of some control variable such as output Tand on the current realization 
of a parameter $\theta_{t}>0$ which is observable by the firm only. Thus there is a problem of adverse selection but not of moral hazard. $\theta_{t}$ has range $\left[\theta^{L}, \theta^{H}\right]$ and is distributed according to the stationary density function $f$. Thus if the firm is in business $\Gamma i t s$ profit function in period $t$ is $\Pi_{t}\left(q_{1}, q_{2}, \ldots, q_{t}, \theta_{t}\right)$ with

$$
\partial^{2} \Pi_{t} / \partial q_{t}^{2} \leq 0 ; \partial^{2} \Pi_{t} / \partial q_{t} \partial \theta_{t} \leq 0 ; \partial \Pi / \partial \theta_{t}<0
$$

If the firm is not in business in $t$ Tit earns zero profit.

An example involving "learning-by-doing" is

$$
\Pi_{t}\left(q_{1}, q_{2}, \ldots, q_{t}, \theta_{t}\right)=p_{t} q_{t} \Leftrightarrow \frac{1}{1+\sum_{s=1}^{t-1} \gamma_{s} q_{s}} C\left(\theta_{t}, q_{t}\right)
$$

where $q \geq 0$ is output $\Gamma p$ is its price and the second term on the right-hand side represents production costs that shift down as cumulative weighted output increases.

The case of a Ricardian non-renewable resource $\Gamma$ with extraction costs increasing as cumulative extraction rises Twould be represented as

$$
\Pi_{t}\left(q_{1}, q_{2}, \ldots, q_{t}, \theta_{t}\right)=p_{t} q_{t} \Leftrightarrow \frac{1}{\beta+\gamma\left[\bar{X} \Leftrightarrow \sum_{s=1}^{t-1} q_{s}\right]} C\left(\theta_{t}, q_{t}\right)
$$

where $\bar{X}, \bar{X} \geq \sum_{s=1}^{t-1} q_{s}$, is the initial amount of non-renewable resource $\Gamma q_{t} \geq 0$ is output $\Gamma$ whose price is $p$ Twhile $\beta$ and $\gamma$ are non negative parameters that specify how extraction costs shift up with cumulative extraction.

As a third example Cconsider a firm whose output is a function $g\left(K_{t}\right)$ of the stock of capital $K_{t}$ Twhose rate of depreciation is $\rho$ एso that $K_{t}=\sum_{\tau=1}^{t} q_{\tau}(1 \Leftrightarrow \rho)^{-(t-\tau)}$. The firm may invest at rate $q_{t} \geq 0$ to build up capital subject to privately observed adjustment costs. This may be represented by

$$
\Pi_{t}\left(q_{1}, q_{2}, \ldots, q_{t}, \theta_{t}\right)=p_{t} g\left(K_{t}\right) \Leftrightarrow C\left(q_{t}, K_{t}, \theta\right)
$$




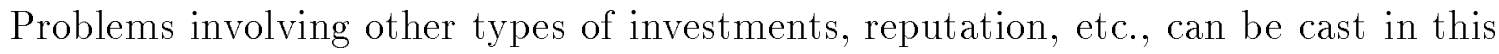
framework. Other technological constraints may be added as needed. For exampleГ a Hotelling mining problem Tinvolving the extraction of a finite stock of homogeneous mineral reserves $\Gamma$ is solved in Gaudet et al. (1991).

In what follows Tin order to avoid useless complications $\Gamma$ we will assume that $q=0$ and $\Pi=0$ when the firm withdraws (shuts down $\Gamma$ either temporarily or permanently). The objective of the firm is to maximize expected cumulative discounted profits $\Gamma$ net of transfers $R_{t}$ to the principal. The latter maximizes a weighted sum consisting of the expected discounted sum of her transfer income on the one hand Tand the expected discounted sum of the net profits left to the firm on the other hand. Whether it be due to the preferences of the principalTor to the cost of transferring income from the firm to the principal $\Gamma$ the latter term is given a lower weight $\Gamma \alpha, 0<\alpha<1 \Gamma$ than the sum of transfers.

The principal is able to commit to a one-period contract but not to any longer-term contract. As already discussed by several authors (seeГfor instanceГLaffont and Tirole (1988)) $\Gamma$ this assumption can capture situations where the principal changes over time $\Gamma$ and the current principal (e.g. government shareholders) cannot commit future ones. It can also arise if long-run contracts are too costly to write or Wwhich is perhaps not very different $\Gamma$ if future actions and contingencies $\Gamma a 1 t h o u g h$ observable upon their realization $\Gamma$ are not easily foreseeable or described $\Gamma$ so that they cannot be used as elements of an enforceable contract. Being unable to precommit Tthe principal cannot credibly give up her claim to a share in future rents in exchange for a lump-sum payment whose amount would be agreed upon before future cost conditions are revealed to the firm. Neither can she credibly commit herself never to resume a severed relationship if it is in her interest to do $\mathrm{so}^{1}$.

\footnotetext{
${ }^{1}$ This last restriction would also follow from renegotiation proofness in a long run contract.
} 


\subsection{Symmetric information}

Consider first the reference scenario of symmetric information. HenceГsuppose that $\theta_{t}$ is observed by both the firm and the principal upon its realization. Then Tat any date $t$ the principal is in a position to require the firm to set to $q_{t}$ so as to maximize total surplus $\Gamma$ the sum of current surplus and the discounted value of expected cumulative future surpluses $\Gamma_{t+1}^{s}\left(q_{t}, \ldots, q_{1}\right)$ Гand to appropriate herself all surpluses (the superscript ${ }^{~} s$ ' refers to symmetric information). Thus in effect $\Gamma$ the principal chooses $q_{t}$ that maximizes

$$
\Pi_{t}\left(q_{t}, \ldots, q_{1}, \theta_{t}\right)+\delta \Gamma_{t+1}^{s}\left(q_{t}, \ldots, q_{1}\right)
$$

Since a total surplus of zero can always be achieved by shutting down forever $\Gamma$ the maximum is necessarily non negative $\Gamma$ and so is $\Gamma_{t+1}^{s}$. However $\Pi_{t}$ may be negative. Future prices Tas well as all functions $C_{t}$ Tare public knowledge; thus the expectation in $\Gamma$ is taken over future values of $\theta$. The conditions for the maximization of (4) must be satisfied at all dates. Thus $\Gamma$ assuming that the price parameters $\Gamma$ the range of $\theta$ Tand the profit functions $\Pi_{t}$ are such that the solution is interior

$$
\frac{\partial \Pi_{t}\left(q_{t}, \ldots, q_{1}, \theta_{t}\right)}{\partial q_{t}}+\delta \frac{\partial \Gamma_{t+1}^{s}\left(q_{t}, \ldots, q_{1}\right)}{\partial q_{t}}=0 \quad \forall \theta_{t} \in\left[\theta^{L}, \theta^{H}\right] \text { and } \forall t
$$

Equation (5) yields $q_{t}$ as a function of $\theta_{t}$ and of the history $\left(q_{t-1}, \ldots, q_{1}\right)$. If it exists $\Gamma$ the terminal period $T^{s}$ is stochastic; for any history when $\Gamma_{t+1}^{s}=0$. If $\Gamma$ for some reason $\Gamma$ before the realization of $\theta_{t} \Gamma$ it is known that $t=T^{s} \Gamma$ then $(5)$ reduces to the static condition

$$
\frac{\partial \prod_{T^{s}}\left(q_{T^{s}}, \ldots, q_{1}, \theta_{T^{s}}\right)}{\partial q_{T^{s}}}=0 \forall \theta_{T^{s}} \in\left[\theta^{L}, \theta^{H}\right]
$$


Denote $q_{T^{s}}^{s}\left(\cdot, \theta_{T^{s}}\right)$ the solution to $(6)$. The corresponding surplus to be transferred to the principal as expected just before the realization of $\theta_{T^{s}}$ Tis

$$
\Gamma_{T^{s}}^{s}\left(q_{T^{s}-1}, \ldots, q_{1}\right)=\int_{\theta^{L}}^{\bar{\theta}_{T^{s}}^{s}}\left[\Pi_{T^{s}}\left(q_{T^{s}}\left(\cdot, \theta_{T^{s}}\right), \ldots, q_{1}, \theta_{T^{s}}\right)\right] f\left(\theta_{T^{s}}\right) d \theta_{T^{s}}
$$

where $f(\theta)$ is the density distribution of $\theta$ and $\bar{\theta}_{T^{s}}^{s}$ is the least efficient profitable type at $T^{s}$. In general $\Gamma$ the next period is not necessarily expected to be the terminal one so that $\Gamma$ in any period $t$ the surplus to be transferred to the principal Tas expected just before the realization of $\theta_{t}$ Tmust include the expected discounted contribution of periods beyond $t$

$$
\Gamma_{t}^{s}\left(q_{t-1}, \ldots, q_{1}\right)=\int_{\theta^{L}}^{\bar{\theta}_{t}^{s}}\left[\Pi_{t}\left(q_{t}^{s}\left(\cdot, \theta_{t}^{s}\right), \ldots, q_{1}, \theta_{t}\right)+\delta \Gamma_{t+1}^{s}\left(q_{t}^{s}\left(\cdot, \theta_{t}^{s}\right), \ldots, q_{1}\right)\right] f\left(\theta_{t}\right) d \theta_{t}
$$

where $q_{t}^{s}$ denotes the solution to $(5)$ at $t$ and $\bar{\theta}_{t}^{s} \Gamma$ the least efficient profitable type at $t \Gamma$ is defined by the condition that expected cumulative discounted profits be at least as high as under the best alternative which is either zero (permanent shutdown) $\Gamma$ or the expected discounted profit from shutting down in $t$ and reopening later on

$$
\bar{\theta}_{t}^{s} \equiv \max \left\{\theta_{t} \mid \Pi\left(q_{t}, \ldots, q_{1}, \theta_{t}\right)+\delta \Gamma_{t+1}^{s}\left(q_{t}, \ldots, q_{1}\right)=\max \left[0, \delta \Gamma_{t+1}^{s}\left(0, q_{t-1}, \ldots, q_{1}\right)\right]\right\}
$$

\subsection{Asymmetric information}

Under asymmetric information the firm observes the realization of $\theta$ at any dateГwhile the principal never does. If Tas assumed $\Gamma$ there is no correlation between the realizations of $\theta$ across periods $\Gamma$ the firm does not lose any of its future informational advantage when it reveals current information to the principal. As a consequence there is no possibility

of a ratchet effect Tand the revelation principle applies as in static setups. Furthermore $\Gamma$ since the principal cannot commit to long-term contracts $\Gamma$ the asymmetric relationship 
is repeated in each period $\Gamma$ but conditioned on an up-dated history.

In the optimal incentive scheme The firm chooses its output and the transfer payment to the principal from a menu offered by the principal and based on the firm's declaration of its type. The construction of such a scheme by standard methods is outlined in the Appendix; by the revelation principle $\Gamma$ the scheme is such that it is not in the interest of the firm to lie about its type so that $\Gamma$ in what follows $\Gamma \theta$ represents the true value $\mathrm{Tas}$ revealed to the principal by the firm. For any history $\left\{q_{t-1}, \ldots, q_{1}\right\} \Gamma$ let $\hat{\Pi}_{t}$ denote net cumulative discounted surplus to the firm

$$
\begin{aligned}
\hat{\Pi}_{t}\left(\theta_{t} ; q_{t-1}, \ldots, q_{1}\right) \equiv & \Pi_{t}\left(q_{t}\left(\theta_{t} ; q_{t-1}, \ldots, q_{1}\right), q_{t-1}, \ldots, q_{1}, \theta_{t}\right) \Leftrightarrow R_{t}\left(\theta_{t} ; q_{t-1}, \ldots, q_{1}\right) \\
& +\delta \Psi_{t+1}\left(q_{t}\left(\theta_{t} ; q_{t-1}, \ldots, q_{1}\right), q_{t-1}, \ldots, q_{1}\right)
\end{aligned}
$$

where $q_{t}\left(\theta_{t} ; q_{t-1}, \ldots, q_{1}\right), R_{t}\left(\theta_{t} ; q_{t-1}, \ldots, q_{1}\right)$ respectively represent production and the transfer under the menu offered by the principal to the firm in period $t$ Tand $\Psi_{t+1}\left(q_{t}\left(\theta_{t} ; q_{t-1}, \ldots, q_{1}\right), q_{t-1}, \ldots, q_{1}\right) \geq 0$ represents the value discounted to $t+1 \Gamma$ as expected at $t$ Tof cumulative future net profits to the firm if it continues its relationship with the principal when it is rational to do so (we specify below conditions under which any firm that stays in business decides to continue the relationship). In order to alleviate the notation $\Gamma$ we shall write $\hat{\Pi}$ and $q$ as functions of $\theta$ only except when more precision is needed. It is shown in the Appendix that

$$
\frac{d \hat{\Pi}_{t}}{d \theta_{t}}=\frac{\partial \Pi_{t}}{\partial \theta_{t}}<0
$$

and

$$
\frac{d q_{t}}{d \theta_{t}} \leq 0
$$

When they correspond to the optimum incentive scheme $\Gamma$ these functions $\Gamma$ and other relevant functions Wwill be denoted with a superscript ' $a$ ' (for asymmetric information). 
At any date $t$ Tfacing the incentive compatibility conditions just outlined $\Gamma$ firms will participate only if their net value is non negative

$$
\hat{\Pi}_{t}\left(\theta_{t}\right) \geq 0 \quad \forall \theta_{t} \in\left[\theta^{L}, \bar{\theta}_{t}\left(q_{t-1}, \ldots, q_{1}\right)\right]
$$

where $\bar{\theta}_{t}$ is the highest-cost firm which participates in period $t$. Note that (10) Tany type $\theta_{t}<\bar{\theta}_{t}$ will participate $\Gamma$ so that (12) may be replaced by

$$
\hat{\Pi}_{t}\left(\bar{\theta}_{t}\right) \geq 0
$$

The assumed inability of the principal to commit herself not to resume a severed relationship if it is not her interest to do so implies that the participation decision is the agent's, and only the agent's. It is impossible for the principal to deny participation to an agent who finds it in his interest

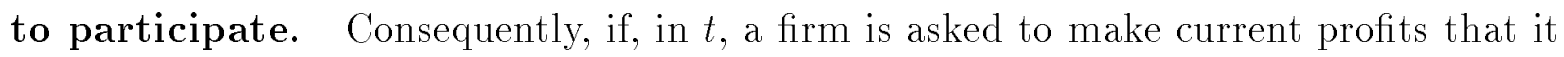
judges too low $\Gamma$ it can always withdraw $\Gamma$ achieve $\Pi_{t}=0$ Tand still participate in future periods if it so wishes. Although the interruption may last any number of periods $\Gamma$ the firm only needs consider the current period $\Gamma$ postponing its next participation decision until the next period. Thus the inability to commit implies a second rationality constraint

$$
\hat{\Pi}_{t}\left(\theta_{t} ; q_{t-1}, \ldots, q_{1}\right) \geq \delta \Psi_{t+1}\left(0, q_{t-1}, \ldots, q_{1}\right) \quad \forall \theta_{t} \in\left[\theta^{L}, \bar{\theta}\left(q_{t-1}, \ldots, q_{1}\right)\right]
$$

In period $t$ Tas shown in the Appendix $\Gamma$ the problem of the principal may be written as

$$
\begin{gathered}
\max _{q_{t}(\cdot), \bar{\theta}_{t}} \int_{\theta^{L}}^{\bar{\theta}_{t}}\left\{\Pi_{t}\left(q_{t}, q_{t-1}, \ldots, q_{1}, \theta_{t}\right) \Leftrightarrow[1 \Leftrightarrow \alpha] \hat{\Pi}_{t}\left(\theta_{t}\right)\right. \\
\left.+\delta\left[\Gamma_{t+1}^{a}\left(q_{t}\left(\theta_{t}\right) ; q_{t-1} \ldots, q_{1}\right)+\Psi_{t+1}^{a}\left(q_{t}\left(\theta_{t}\right) ; q_{t-1} \ldots, q_{1}\right)\right]\right\} f\left(\theta_{t}\right) d \theta_{t}
\end{gathered}
$$

subject to $(10) \Gamma(13) \Gamma$ and $(14) \Gamma$ where $\Gamma_{t+1}^{a}$ denotes cumulative future transfers to the 
principal $\Gamma$ discounted to $t+1$ Tas expected at $t$ Tand the superscripts ${ }^{~} a$ ' in $\Gamma_{t+1}^{a}$ and $\Psi_{t+1}^{a}$ indicate that both the agent and the principal know that the principal will find it in her interest to use an incentive mechanism in all remaining periods. Treating $\hat{\Pi}_{t}\left(\theta_{t}\right)$ as a state variable and $q_{t}\left(\theta_{t}\right)$ as a control variable $(15)$ may be solved as an optimal control problem (see the Appendix). The solution $q_{t}^{a}\left(\theta_{t} ; q_{t-1}, \ldots, q_{1}\right)$ must be such that

$$
\frac{\partial \Pi_{t}\left(q_{t}, \ldots, q_{1}, \theta_{t}\right)}{\partial q_{t}}+[1 \Leftrightarrow \alpha] \frac{F\left(\theta_{t}\right)}{f\left(\theta_{t}\right)} \frac{\partial^{2} \Pi_{t}\left(q_{t}, \ldots, q_{1}, \theta_{t}\right)}{\partial \theta_{t} \partial q_{t}}=\Leftrightarrow \delta \frac{\partial S_{t+1}^{a}\left(q_{t}, \ldots, q_{1}\right)}{\partial q_{t}}
$$

where $S_{t+1}^{a} \equiv \Gamma_{t+1}^{a}+\Psi_{t+1}^{a}$ represents the cumulative surplus $\Gamma$ discounted to $t+1 \Gamma$ as expected at $t$.

Also $\Gamma$ at $\bar{\theta}_{t}^{a}$ Tconstraints (13) and (14) must be satisfied. Suppose that the binding one is $(13)$ Tthen

$$
\hat{\Pi}_{t}\left(\bar{\theta}_{t}^{a} ; q_{t-1}, \ldots, q_{1}\right)=0
$$

This is the usual static result (Fact D) that the marginal participating firm is left without any rent. However this result does not hold in general: suppose now that the binding participation constraint is (14); then

$$
\hat{\Pi}_{t}\left(\bar{\theta}_{t}^{a} ; q_{t-1}, \ldots, q_{1}\right)=\delta \Psi_{t+1}^{a}\left(0, q_{t-1}, \ldots, q_{1}\right)>0
$$

Here Tunlike the static case Tthe marginal participating firm is left with a strictly positive rent; otherwise it would be better off temporarily withdrawing from its relationship with the principal. While $\Psi_{t+1}^{a}\left(q_{t}^{a}, q_{t-1}, \ldots, q_{1}\right)$ is positive by definition, it is possible for $\Psi_{t+1}^{a}\left(0, q_{t-1}, \ldots, q_{1}\right)$ to be non positive; but (18) is not binding in that case. Since $\hat{\Pi}_{t} \Leftrightarrow \delta \Psi_{t+1}^{a}=\Pi_{t} \Leftrightarrow R_{t} \Gamma(18)$ is binding whenever it would be desirable for the principal to ask the firm to experience a negative cash flow in the current period, in exchange for positive expected future profits. For example, in an investment context, whenever future output prices are high relative to the 
acquisition cost of equiment, it is desirable, in a full information situation, to build up capital, thus incurring negative current cash flows. In such a situation, the inability to commit requires the principal to set $R_{t}<0$ if the firm is to agree to current investment expenditures involving a negative $\Pi_{t}$. Combining the above two possible terminal conditions $\Gamma$ the participation margin under asymmetric information (analog to (9) under symmetry) is

$$
\bar{\theta}_{t}^{a}=\max \left\{\theta_{t} \mid \hat{\Pi}\left(\theta_{t} ; q_{t-1}, \ldots, q_{1}\right)=\max \left[0, \delta \Psi_{t+1}^{a}\left(0, q_{t-1}, \ldots, q_{1}\right)\right]\right\}
$$

The foregoing discussion implies the proposition below

Proposition 1 When $\Psi_{t+1}^{a}\left(0, q_{t-1}, \ldots, q_{1}\right)>0$, the marginal participating firm enjoys a strictly positive rent.

\section{Comparing the static model with the dynamic model}

We have just shown that Fact 2 - zero rent to the marginal participating firm - no longer holds in our dynamic setup. We are now ready for a more thorough comparison of the solutions under symmetric Tand under asymmetric Tinformation.

Note first that the static solution obtains as a special case of the model studied in the previous section. Indeed $\Gamma$ if $T=T^{a}=T^{s}$ is the last period $\Gamma$ the solution under symmetric information is given by (6) taken at $T$ Tor

$$
\frac{\partial \Pi_{T}\left(q_{T}, \ldots, q_{1}, \theta_{T}\right)}{\partial q_{T}}=0 \quad \forall \theta_{T} \in\left[\theta^{L}, \theta^{H}\right]
$$


The solution under asymmetric information is given by (16) taken at $t=T$ Tor

$$
\frac{\partial \Pi_{T}\left(q_{T}, \ldots, q_{1}, \theta_{T}\right)}{\partial q_{T}}+[1 \Leftrightarrow \alpha] \frac{F\left(\theta_{T}\right)}{f\left(\theta_{T}\right)} \frac{\partial^{2} \Pi_{T}\left(q_{T}, \ldots, q_{1}, \theta_{T}\right)}{\partial \theta_{T} \partial q_{T}}=0
$$

One notes that the term in $S_{t}^{a}$ which appears in (16) has vanished since expected future surplus is identically zero in the last period. Comparing (21) with (20) Tone can verify that the solutions coincide for the 'best' types $\left(\theta_{T}=\theta^{L}\right) \Gamma$ provided output histories are identical up to $T \Leftrightarrow 1$. This illustrates Fact 3. Given (1) $\Gamma$ if the hazard rate $\frac{F(\theta)}{f(\theta)}$ is positively monotonous $\Gamma$ the solution is clearly unique and strictly decreasing in $\theta_{T}$ Twhich implies that it is separating over $\left[\theta^{L}, \bar{\theta}_{T}\right]$. This $\Gamma$ together with $(10)$ Cillustrates Fact 4 . Note that pooling trivially occurs (with $q_{T}=0$ ) for $\theta_{T} \in\left[\bar{\theta}_{T}, \theta^{H}\right]$.

Turning to the dynamic case $\Gamma$ we note that $\Gamma$ since the right-hand side of $(16)$ is independent of $\theta_{t}$ Tthe same condition (monotonous hazard rate) which ensures that the solution is fully separating for types $\theta_{t} \leq \bar{\theta}_{t}$ in the static case (Fact 4) Timplies separation here. Also $\Gamma$ for $\theta_{t} \leq \bar{\theta}_{t}$ Tsince (11) holds at arbitrary dates $\Gamma$ the second feature of Fact 4 (monotonicity of $q$ and $\hat{\Pi}$ ) survives the extension to a dynamic setup. HoweverTto the extent that $\bar{\theta}_{t}$ depends on future conditions and that firms which do not participate in $t$ may participate again later on Tit would be misleading to describe the solution as fully separating. Rather $\Gamma$ the solution is separating for $\theta_{t} \leq \bar{\theta}_{t}$ Tand involves pooling at $q_{t}=0$ for $\theta_{t}>\bar{\theta}_{t}$. Thus pooling occurs whenever $\bar{\theta}_{t}<\theta^{H}$. This is summarized in the next proposition.

Proposition 2 If $\frac{F(\theta)}{f(\theta)}$ is positively monotonous, the solution is fully separating over $\left[\theta^{L}, \bar{\theta}_{t}\right]$ and such that $q_{t}^{a}\left(q_{t-1}, \ldots, q_{1}, \theta_{t}\right)$ and $\hat{\Pi}_{t}\left(q_{t-1}, \ldots, q_{1}, \theta_{t}\right)$ are strictly decreasing in $\theta_{t}$. Over $\left[\bar{\theta}_{t}, \theta^{H}\right]$, there is pooling at $q_{t}^{a}\left(q_{t-1}, \ldots, q_{1}, \theta_{t}\right)=0$.

We now focus on the dynamic counterpart of Fact 3; thus we want to compare output under asymmetric information with output under symmetric information at arbitrary 
dates $\Gamma a n d$ determine whether the 'best' type selects the sameГefficient $\Gamma$ level of $q_{t}$ under both informational setups. For interior solutions $\Gamma$ this means that condition (16) Гnamely

$$
\frac{\partial \Pi_{t}\left(q_{t}, \ldots, q_{1}, \theta_{t}\right)}{\partial q_{t}}+[1 \Leftrightarrow \alpha] \frac{F\left(\theta_{t}\right)}{f\left(\theta_{t}\right)} \frac{\partial^{2} \Pi_{t}\left(q_{t}, \ldots, q_{1}, \theta_{t}\right)}{\partial \theta_{t} \partial q_{t}}=\Leftrightarrow \delta \frac{\partial S_{t+1}^{a}\left(q_{t}, \ldots, q_{1}\right)}{\partial q_{t}}
$$

has to be compared with condition (5) Tnamely

$$
\frac{\partial \prod_{t}\left(q_{t}, \ldots, q_{1}, \theta_{t}\right)}{\partial q_{t}}=\Leftrightarrow \delta \frac{\partial \Gamma_{t+1}^{s}\left(q_{t}, \ldots, q_{1}\right)}{\partial q_{t}}
$$

Comparing these conditions for identical output histories $\Gamma$ we see that a necessary and sufficient condition for types $\theta^{L}$ to choose the same output under symmetry as under asymmetry is

$$
\frac{\partial S_{t+1}^{a}\left(q_{t}, \ldots, q_{1}\right)}{\partial q_{t}}=\frac{\partial \Gamma_{t+1}^{s}\left(q_{t}, \ldots, q_{1}\right)}{\partial q_{t}}
$$

The condition requires the marginal impact of increasing output on total expected future surpluses from continuing the relationship under asymmetric information to equal the marginal impact of increasing output on total expected future surpluses under symmetric information. This result is stated in the following proposition.

Proposition 3 Firms of type $\theta^{L}$ choose a different output under asymmetric information than under symmetric information, unless $\frac{\partial S_{t+1}^{a}\left(q_{t}, \ldots, q_{1}\right)}{\partial q_{t}}=\frac{\partial \Gamma_{t+1}^{s}\left(q_{t}, \ldots, q_{1}\right)}{\partial q_{t}}$

It is well known that the revelation of private information is achieved at a cost in efficiency $\Gamma$ so that $\Gamma$ generally $\Gamma S_{t+1}^{a} \neq \Gamma_{t+1}^{s}$. Indeed $\Gamma$ Fact 3 identifies type $\theta^{L}$ as the one exception to that rule in a static setup. In a dynamic context $\Gamma$ however $\Gamma$ a firm of type $\theta_{t}=\theta^{L}$ will be of a higher-cost type with a positive probability in some future period $\Gamma$ so that its expected future surplus under asymmetry is certainly lower than under symmetry. While it does not follow that (22) is necessarily violated is a condition on marginal Trather than total $\Gamma$ surpluses $\Gamma$ the presumption is that it will 
be violated in most circumstances.

We have shown that the static properties of static incentive contracts $\Gamma$ in particular Fact 2 (the marginal participating firm is left without any rent) and Fact 3 (the 'best' firm is asked to operate efficiently) no longer hold in our dynamic setup. Unlike previously studied dynamic asymmetric information models $\Gamma$ where the dynamic arises from intertemporal correlations between the privately observed parameter rather than through the impact of past decisions on current profits $\Gamma$ this happens despite the fact that the revelation principle still applies. The optimum contracts now involve future expected surpluses. If these are high enough even when the firm does not participate in the current period $\Gamma$ then the static rationality constraint (zero profit) no longer ensures participation: the firm faces a strictly positive best alternative Wwhich explains why Fact Dno longer holds. The relevance of future expected surpluses to current output decisions also implies that the 'best' firm itself is affected by asymmetry in future information despite our assumption that there is no correlation between stochastic realizations from one period to the other: while the firm may be of type $\theta^{L}$ in the current period type in future periods will be worse with a positive probability surpluses associated with type $\theta^{L}$ will be lower than under symmetric information. This is why Fact 3 no longer holds.

\section{An example}

Do these findings have any importance for theoretical modeling of dynamic incentive contracts? Perhaps surprisingly the example below will show that exceptions may arise under assumptions that are widely accepted in dynamic asymmetric information models. Consider the cost of adjustment model mentioned at the beginning of the paper. As is

customary in the information literature Tassume a fixed number of periods and a range of $\theta$ such that $\Gamma$ considering prices $\Gamma a 11$ types participate under both informational contexts: 
$\bar{\theta}_{t}^{s}=\bar{\theta}_{t}^{a}=\theta^{H}$. Thus we have $\Gamma$ for a firm in business $\Gamma$ whose current stock of capital is $K_{t}=\sum_{1}^{t} q_{\tau}(1 \Leftrightarrow \rho)^{t-\tau}$

$$
\Pi_{t}\left(q_{1}, q_{2}, \ldots, q_{t}, \theta_{t}\right)=p_{t} g\left(K_{t}\right) \Leftrightarrow C\left(q_{t}, K_{t}, \theta\right)
$$

with $q_{t} \geq 0, K_{t} \geq 0 \Gamma K_{0}>0$ given $^{2}$. Assume further ${ }^{3}$ that $C=\frac{1}{2} q_{t}^{2}+\left(\theta_{t}+\phi_{t}\right) q_{t}+\theta_{t}$ and that

$$
\begin{gathered}
p_{t}=p \text { for } t \leq T \\
p_{t}=0 \text { afterwards } \\
\theta_{t} \sim f\left(\theta_{t}\right), \theta_{t} \in\left[\theta^{L}, \theta^{H}\right] \text { for } t \leq T \\
\theta_{t}=0 \text { for } t>T \\
\phi_{t}=\phi>0 \text { for } t \leq T \\
\phi_{t}=0 \text { afterwards }
\end{gathered}
$$

The assumptions on $p_{t}, \theta_{t}$, and $\phi_{t}$ ensure that no profitable production or investment activity may take place after $T$ Sso that the fixed horizon would in fact be endogenously chosen.

Let us consider a firm whose capital is $K_{t}$ under symmetric and asymmetric information alternatively. Under symmetric information $\Gamma$ if $t$ is the second last period $\Gamma$ so that $t+1=T \Gamma$

$$
\Gamma_{t+1}^{s}=\Gamma_{T}^{s}=\int_{\theta^{L}}^{\bar{\theta}_{t+1}^{s}}\left\{p_{t+1} g\left(K_{t+1}^{s}\right) \Leftrightarrow \frac{1}{2}\left[q_{t+1}^{s}\right]^{2} \Leftrightarrow\left[\theta_{t+1}+\phi_{t+1}\right] q_{t+1}^{s} \Leftrightarrow \theta_{t+1}\right\} f\left(\theta_{t+1}\right) d \theta_{t+1}
$$

\footnotetext{
${ }^{2}$ Using the convention that current investment is incorporated into current capital immediately rather than with a lag of one period allows us to illustrate our point more compactlyrby considering the second last period rather than period $T-2$.

${ }^{3}$ Allowing $\theta$ to enter as a fixed cost $\Gamma$ besides affecting the marginal cost $\Gamma$ is not customary in the principal-agent literature. We use this formulation as a simple way to ensure that assumption $\frac{\partial \pi}{\partial \theta}<0$ is met. This assumption could itself be weakened to allow equality complexities would only obscure the foregoing argument. In Gaudet et al. (1992) Twe solve a problem where $q$ may take any real value $\Gamma$ the fixed cost is observable $\Gamma$ and $\frac{\partial \pi}{\partial \theta} \leq 0$.
} 
The interior solution for $q_{t}^{s}$ is given by (5) Гwhich becomes

$$
\Leftrightarrow p_{t}+q_{t}^{s}+\theta_{t}+\phi_{t}=\delta p_{t+1} \int_{\theta^{L}}^{\bar{\theta}_{t+1}^{s}} \frac{\partial g\left(K_{t}[1 \Leftrightarrow \rho]+q_{t+1}^{s}\right)}{\partial q_{t}} f\left(\theta_{t+1}\right) d \theta_{t+1}
$$

Similarly Tunder asymmetric information $\Gamma$ if $t$ is the second last period

$$
S_{t+1}^{a}=S_{T}^{a}=\int_{\theta^{L}}^{\bar{\theta}_{t+1}^{a}}\left\{p_{t+1} g\left(K_{t+1}^{a}\right) \Leftrightarrow \frac{1}{2}\left[q_{t+1}^{a}\right]^{2} \Leftrightarrow\left[\theta_{t+1}+\phi_{t+1}\right] q_{t+1}^{a} \Leftrightarrow \theta_{t+1}\right\} f\left(\theta_{t+1}\right) d \theta_{t+1}
$$

The interior solution for $q_{t}^{a}$ must satisfy (16) $\Gamma$ which gives

$$
\Leftrightarrow p_{t}+q_{t}^{a}+\theta_{t}+\phi_{t} \Leftrightarrow[1 \Leftrightarrow \alpha] \frac{F\left(\theta_{t}\right)}{f\left(\theta_{t}\right)}=\delta p_{t+1} \int_{\theta^{L}}^{\bar{\theta}_{t+1}^{a}} \frac{\partial g\left(K_{t}[1 \Leftrightarrow \rho]+q_{t+1}^{a}\right)}{\partial q_{t}} f\left(\theta_{t+1}\right) d \theta_{t+1}
$$

Thus $\Gamma$ in period $t=T \Leftrightarrow 1 \Gamma$ for $\theta_{t}=\theta^{L} \Gamma$ the left-hand sides of (23) and (24) are equal $\Gamma$ except $\Gamma$ possibly $\Gamma$ for differences in $q_{t}^{a}$ and $q_{t}^{s} \Gamma$ so that

$$
\begin{aligned}
q_{t}^{a}<(\geq) q_{t}^{s} & \Leftrightarrow \\
& \int_{\theta^{L}}^{\bar{\theta}_{t+1}^{a}} \frac{\partial}{\partial q_{t}^{a}} g\left(K_{t}[1 \Leftrightarrow \rho]+q_{t+1}^{a}\right) f\left(\theta_{t+1}\right) d \theta_{t+1}<(\geq) \int_{\theta^{L}}^{\bar{\theta}_{t+1}^{s}} \frac{\partial}{\partial q_{t}^{s}} g\left(K_{t}[1 \Leftrightarrow \rho]+q_{t+1}^{s}\right) f\left(\theta_{t+1}\right) d \theta_{t+1}
\end{aligned}
$$

The above integrals are likely to differ: first $\Gamma \bar{\theta}_{t+1}^{a}$ normally differs from $\bar{\theta}_{t+1}^{s}$; second $\Gamma$ $q_{t+1}^{a}$ will differ from $q_{t+1}^{s}$ for all realizations of $\theta_{t+1}$ but $\theta^{L}$. However $\Gamma$ allowing for the assumption that $\bar{\theta}^{a}=\bar{\theta}^{s}=\theta^{H} \Gamma$ and noting that $\frac{\partial K_{t}}{\partial q_{t}}=1$, equality obtains if $\frac{\partial g\left(K_{t}[1-\rho]+q_{t+1}^{a}\right)}{\partial q_{t}}=\frac{\partial g\left(K_{t}[1-\rho]+q_{t+1}^{s}\right)}{\partial q_{t}}$ for all $\theta_{t+1} \Gamma$ which is true if $\frac{\partial g}{\partial K}$ is independent of $K$ and $q$ i.e. if the production technology exhibits constant returns.

This exception to the general results presented above arises because $\Gamma$ with a constant returns technology $\Gamma$ the marginal impact of changing $q_{t}$ on expected future surplus is independent of the future level of $K$ provided the participation margin is unaffected. Indeed $\Gamma$ constant returns is also necessary in this example: if $g$ is not linear $\Gamma$ the integral on the right-hand side of (24) will differ from the corresponding integral in (23) unless 
$q_{t+1}^{a}\left(\theta_{t+1}\right)=q_{t+1}^{s}\left(\theta_{t+1}\right)$ for all $\theta_{t+1}$; but such equality itself requires $g$ to be linear as can be verified by comparing the appropriate versions of (23) and (24). As our example clearly illustrates $\Gamma$ however $\Gamma$ the linearity of the current profit function in $K_{t}$ is not sufficient to ensure the linearity of cumulative expected future surpluses in $q_{t}$. The participation margins $\bar{\theta}_{t}^{a}$ and $\bar{\theta}_{t}^{s}$ must also be identical and invariant; this is the case when they arise as corner solutions as we have assumed in our example ${ }^{4}$.

We should note also that it is easy $\Gamma$ although cumbersome $\Gamma$ to extend the analysis of our example to earlier periods. Besides the conditions just identified $\Gamma$ the survival of Fact 3 will obtain if $\Gamma$ for each supplementary period $\Gamma$ the participation margin in the new period is a corner solution at $\theta^{H}$. Consequently ffor the cost of adjustment model $\Gamma$ Fact 3 may arise under apparently benign assumptions and $\Gamma$ perhaps $\Gamma$ be taken for what it is not: a generic property of the type of dynamic models studied in this paper. The reader can verify that no such possibility arises with the other two examples (learning by doing; resource extraction) mentioned earlier.

\footnotetext{
${ }^{4}$ The assumption that $\bar{\theta}_{t}^{s}=\bar{\theta}_{t}^{a}=\theta^{H}$ is satisfied if and only ifTat any date $\max \left\{\theta_{t} \mid \Pi\left(q_{t}, \ldots, q_{1}, \theta_{t}\right)+\delta \Gamma_{t+1}^{s}\left(q_{t}, \ldots, q_{1}\right) \geq \max \left\{0, \delta \Gamma_{t+1}^{s}\left(0, q_{t-1}, \ldots, q_{1}\right)\right\}\right\}=\theta^{H}$ and $\max \left\{\theta_{t} \mid \hat{\Pi}\left(\theta_{t} ; q_{t-1}, \ldots, q_{1}\right) \geq \max \left[0, \delta \Psi_{t+1}^{a}\left(0, q_{t-1}, \ldots, q_{1}\right)\right]\right\}=\theta^{H}$ In particular $\Gamma$ when $t=T-1$ and $g$ is linear (say $g(k)=K$ ) $\Gamma$ these assumptions reduce to $\max \left\{\theta_{t} \mid p K_{t}-q_{t}^{2}-\left(\theta_{t}+\phi\right) q_{t}-\theta_{t} \geq 0\right\}=\theta^{H}$ and$$
\max \left\{\theta_{t} \mid p K_{t}-q_{t}^{2}-\left(\theta_{t}+\phi\right) q_{t}-\theta_{t}-R_{t} \geq 0\right\}=\theta^{H}
$$

They are obviously satisfied when $p$ and $K_{t}$ are high enough relative to $\theta^{H}$ and $\phi$.
} 


\section{Appendix: Asymmetric information}

\section{Incentive compatibility}

Denote the net total value of the firm at $t$

$$
\begin{aligned}
& \phi_{t}\left(\tilde{\theta}_{t}, \theta_{t} ; q_{t-1}, \ldots, q_{1}\right) \equiv \Pi_{t}\left(q_{t}\left(\tilde{\theta}_{t} ; q_{t-1}, \ldots, q_{1}\right), \ldots, q_{1}, \theta_{t}\right) \\
& \Leftrightarrow R_{t}\left(\tilde{\theta}_{t} ; q_{t-1}, \ldots, q_{1}\right)+\delta \Psi_{t+1}\left(q_{t}\left(\tilde{\theta}_{t} ; q_{t-1}, \ldots, q_{1}\right), \ldots, q_{1}\right)
\end{aligned}
$$

where $q_{t}\left(\tilde{\theta}_{t} ; q_{t-1}, \ldots, q_{1}\right), R_{t}\left(\tilde{\theta}_{t} ; q_{t-1}, \ldots, q_{1}\right)$ respectively represent production and the transfer under the menu offered by the principal to the firm in period $t$ Tand

$\Psi_{t+1}\left(q_{t}\left(\tilde{\theta}_{t} ; q_{t-1}, \ldots, q_{1}\right), \ldots, q_{1}\right) \geq 0$ represents the value discounted to $t+1$ Tas expected at $t$ Tof cumulated future net profits to the firm if it continues its relationship with the principal (we will later on impose conditions under which it is in the interest of the firm to do so). When they correspond to the optimum incentive schemeTthese functions will be denoted with a superscript ' $a$ '. We will also drop arguments $q_{t-1}, \ldots, q_{1}$ in order to alleviate notation.

Incentive compatibility is defined by the condition that the firm chooses to reveal its true type when selecting an output-transfer combination from the menu. Thus $\tilde{\theta}_{t}$ must equal $\theta_{t}$ when the firm chooses it so as to maximize $\phi_{t}$. The first-order condition for period $t \Gamma \partial \phi_{t} / \partial \tilde{\theta}_{t}=0 \Gamma$ must be satisfied when $\tilde{\theta}_{t}=\theta_{t}$ for all $\theta_{t}^{5}$

$$
\left[\frac{\partial \prod_{t}\left(q_{t}\left(\tilde{\theta}_{t}\right), \ldots, q_{1}, \theta_{t}\right)}{\partial q_{t}}+\delta \frac{\partial \Psi_{t+1}\left(q_{t}\left(\tilde{\theta}_{t}\right), \ldots, q_{1}\right)}{\partial q_{t}}\right] \frac{d q_{t}\left(\tilde{\theta}_{t}\right)}{d \tilde{\theta}_{t}}=\frac{d R\left(\tilde{\theta}_{t}\right)}{d \tilde{\theta}_{t}}
$$

when $\tilde{\theta}_{t}=\theta_{t} \forall \theta_{t} \in\left[\theta^{L}, \theta^{H}\right]$.

\footnotetext{
${ }^{5}$ Clearly existence and unicity require some additional conditions on $\Pi$ which we assume to be satisfied.
} 
For any variations $d \theta_{t}=d \tilde{\theta}_{t} \Gamma(25)$ must continue to hold. This $\Gamma$ together with the second-order condition $\partial^{2} \phi_{t+1} / \partial \tilde{\theta}_{t}^{2} \leq 0$ implies

$$
\frac{\partial^{2} \Pi_{t}}{\partial q_{t} \partial \theta_{t}} \frac{d q_{t}}{d \tilde{\theta}_{t}} \geq 0
$$

Since $\partial^{2} \Pi / \partial q_{t} \partial \theta_{t} \leq 0$ (abandoning from here on the distinction between $\tilde{\theta}_{t}$ and $\theta_{t}$ since they are equal) $\Gamma \frac{d q_{t}}{d \theta_{t}} \leq 0 \Gamma$ which is (11). Also $\Gamma$ since by definition $\hat{\Pi}_{t}\left(\theta_{t}\right)=\phi_{t}\left(\theta_{t}, \theta_{t}\right) \Gamma$ applying the envelope theorem $\Gamma$ we have (10): $\frac{d \hat{\Pi}_{t}}{d \theta_{t}}=\frac{\partial \Pi_{t}}{\partial \theta_{t}}<0$.

\section{The optimal contract}

The principal must choose the functions $q_{t}(\cdot)$ and $R_{t}(\cdot)$ Tand the cutoff type $\bar{\theta}_{t}$ so as to maximize

$\int_{\theta^{L}}^{\bar{\theta}_{t}}\left\{R_{t}\left(\theta_{t}\right)+\alpha\left[\hat{\Pi}_{t}\left(\theta_{t}\right) \Leftrightarrow \delta \Psi_{t+1}^{a}\left(q_{t}, \ldots, q_{1}\right)\right]+\delta\left[\Gamma_{t+1}^{a}\left(q_{t}, \ldots, q_{1}\right)+\alpha \Psi_{t+1}^{a}\left(q_{t}, \ldots, q_{1}\right)\right]\right\} f\left(\theta_{t}\right) d \theta_{t}$

where $\Gamma_{t+1}$ denotes cumulative future transfers to the principalГdiscounted to $t+1$ Tas expected at $t$ and the superscripts in $\Gamma_{t+1}^{a}$ and $\Psi_{t+1}^{a}$ indicate that the agent knows that the principal will find it in her interest to use an incentive mechanism in all remaining periods. Types $\theta_{t}>\bar{\theta}_{t}$ are not necessarily out of business; they are just pooled in period $t$.

Eliminating $R_{t}$ using the definitions of $\phi_{t}$ and $\hat{\Pi}_{t} \Gamma$ the problem becomes $(15)$

$$
\begin{gathered}
\max _{q_{t}(\cdot), \bar{\theta}_{t}} \int_{\theta^{L}}^{\bar{\theta}_{t}}\left\{\Pi_{t}\left(q_{t}\left(\theta_{t}\right), q_{t-1}, \ldots, q_{1}, \theta_{t}\right) \Leftrightarrow[1 \Leftrightarrow \alpha] \hat{\Pi}_{t}\left(\theta_{t}\right)\right. \\
\left.+\delta\left[\Gamma_{t+1}^{a}\left(q_{t}\left(\theta_{t}\right) ; q_{t-1} \ldots, q_{1}\right)+\Psi_{t+1}^{a}\left(q_{t}\left(\theta_{t}\right) ; q_{t-1} \ldots, q_{1}\right)\right]\right\} f\left(\theta_{t}\right) d \theta_{t}
\end{gathered}
$$


subject to $(10) \Gamma(13)$ Tand (14). The Hamiltonian is

$$
\begin{gathered}
H=\left\{\Pi_{t}\left(q_{t}\left(\theta_{t}\right), \ldots, q_{1}, \theta_{t}\right) \Leftrightarrow[1 \Leftrightarrow \alpha] \hat{\Pi}_{t}\left(\theta_{t}\right)+\delta S_{t+1}^{a}\left(q_{t}\left(\theta_{t}\right), \ldots, q_{1}\right)\right\} f\left(\theta_{t}\right) \\
+\mu_{t} \frac{\partial \Pi_{t}\left(q_{t}\left(\theta_{t}\right), \ldots, q_{1}, \theta_{t}\right)}{\partial \theta_{t}}+\lambda_{t}\left[\hat{\Pi}_{t}\left(\theta_{t}\right) \Leftrightarrow \delta \Psi_{t+1}^{a}\left(0, q_{t-1}, \ldots, q_{1}\right)\right]
\end{gathered}
$$

where $\mu_{t}$ is the costate variable associated with $\hat{\Pi}_{t}, \lambda_{t} \geq 0$ is a multiplier associated with (14) Гand $S_{t+1}^{a}$ is the expected future surplus $\Gamma$ defined as the sum of $\Gamma_{t+1}^{a}$ and $\Psi_{t+1}^{a}$.

The following necessary conditions must hold

$$
\begin{gathered}
\left\{\frac{\partial \Pi_{t}\left(q_{t}, \ldots, q_{1}, \theta_{t}\right)}{\partial q_{t}}+\delta \frac{\partial S_{t+1}^{a}\left(q_{t}, \ldots, q_{1}\right)}{\partial q_{t}}\right\} f\left(\theta_{t}\right)+\mu_{t} \frac{\partial^{2} \Pi_{t}\left(q_{t}, \ldots, q_{1}, \theta_{t}\right)}{\partial \theta_{t} \partial q_{t}}=0 \\
\frac{d \mu_{t}}{d \theta_{t}}=[1 \Leftrightarrow \alpha] f\left(\theta_{t}\right) \Leftrightarrow \lambda_{t}\left(\theta_{t}\right)
\end{gathered}
$$

Also $\Gamma$ by $(10) \Gamma \hat{\Pi}_{t}\left(\theta^{L}\right)$ necessarily satisfies $(13)$ if so does $\hat{\Pi}_{t}\left(\bar{\theta}_{t}\right)$; consequently $\hat{\Pi}_{t}\left(\theta^{L}\right)$ is free $\Gamma$ so that

$$
\mu_{t}\left(\theta^{L} ; q_{t-1}, \ldots, q_{1}\right)=0
$$

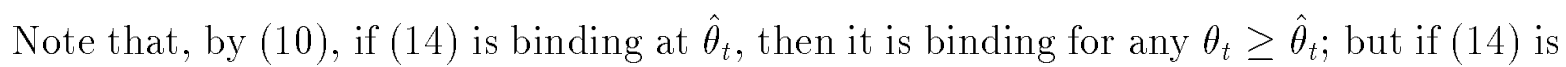
binding over $\left[\hat{\theta}_{t}, \bar{\theta}_{t}\right] \Gamma$ then $\hat{\Pi}$ is constant over that interval $\Gamma$ contradicting (10). It follows that (14) may be binding only at $\theta_{t}=\bar{\theta}_{t}$ if at all. As a result $\Gamma \lambda_{t}=0 \forall \theta_{t}<\bar{\theta}_{t}$ so that $\Gamma$ integrating (28) considering (29) Гwe have

$$
\mu_{t}\left(\theta_{t} ; q_{t-1}, \ldots, q_{1}\right)=[1 \Leftrightarrow \alpha] F\left(\theta_{t}\right)
$$

Substituting for $\mu_{t}$ in (27) $\Gamma$ we obtain (16)

$$
\frac{\partial \Pi_{t}\left(q_{t}, \ldots, q_{1}, \theta_{t}\right)}{\partial q_{t}}+[1 \Leftrightarrow \alpha] \frac{F\left(\theta_{t}\right)}{f\left(\theta_{t}\right)} \frac{\partial^{2} \Pi_{t}\left(q_{t}, \ldots, q_{1}, \theta_{t}\right)}{\partial \theta_{t} \partial q_{t}}=\Leftrightarrow \delta \frac{\partial S_{t+1}^{a}\left(q_{t}, \ldots, q_{1}\right)}{\partial q_{t}}
$$

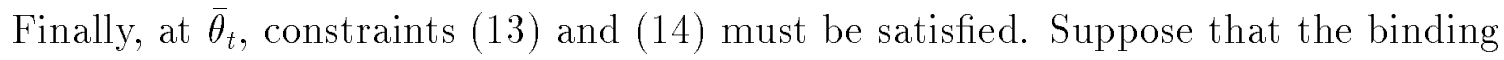


one is (13); then the transversality condition is $\mu_{t}\left(\bar{\theta}_{t}\right) \hat{\Pi}_{t}\left(\bar{\theta}_{t} ; q_{t-1}, \ldots, q_{1}\right)=0$ so that $\Gamma$ since $\mu_{t}\left(\bar{\theta}_{t}\right)>0$, we obtain $(17)$

$$
\hat{\Pi}_{t}\left(\bar{\theta}_{t} ; q_{t-1}, \ldots, q_{1}\right)=0
$$

Suppose now that the binding participation constraint is (14); then we have (18)

$$
\hat{\Pi}_{t}\left(\bar{\theta}_{t} ; q_{t-1}, \ldots, q_{1}\right)=\delta \Psi_{t+1}^{a}\left(0, q_{t-1}, \ldots, q_{1}\right)>0
$$




\section{References}

BaronГD. P. (1989) "Design of Regulatory Mechanisms and Institutions" in Handbook of Industrial OrganisationTed. R. Schmalensee and R. D. Willig. New York: North Holland $\Gamma 1347-1447$.

BesankoГD. and D. E. M. Sappington (1987) Designing Regulatory Policy with Limited Information. London: Harwood Academic Publishers.

GaudetГG.ГP. Lasserre and N. V. Long (1995) "Optimal Resource Royalties with Unknown and Temporally Independent Extraction Cost Structures I" International Economic Review $\Gamma$ forthcoming $\Gamma$.

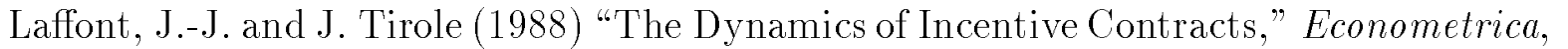
$56 \Gamma 1153-1175$. 